\title{
Physical properties of model biological lipid bilayers: insights from all-atom molecular dynamics simulations
}

\author{
Ganesh Shahane ${ }^{1} \cdot$ Wei Ding ${ }^{2} \cdot$ Michail Palaiokostas ${ }^{2} \cdot$ Mario Orsi $^{3}$ (D)
}

Received: 19 July 2018 / Accepted: 7 February 2019 / Published online: 26 February 2019

(C) The Author(s) 2019

\begin{abstract}
The physical properties of lipid bilayers are sensitive to the specific type and composition of the lipids that make up the many different types of cell membranes. Studying model bilayers of representative heterogeneous compositions can provide key insights into membrane functionality. In this work, we use atomistic molecular dynamics simulations to characterize key properties in a number of bilayer membranes of varying composition. We first examine basic properties, such as lipid area, volume, and bilayer thickness, of simple, homogeneous bilayers comprising several lipid types, which are prevalent in biological membranes. Such lipids are then used in simulations of heterogeneous systems representative of bacterial, mammalian, and cancer membranes. Our analysis is especially focused on depth-dependent, transmembrane profiles; in particular, we calculate lateral pressure and dipole potential profiles, two fundamental properties which play key roles in a large number of biological functions.
\end{abstract}

Keywords Molecular dynamics simulations $\cdot$ Lipid bilayer $\cdot$ Lateral pressure $\cdot$ Dipole potential

\section{Introduction}

Lipid bilayers are fundamental structures in biology; they form a continuous barrier around cells, compartmentalize the intracellular space, act as selective barriers for the permeation of molecules, and also play key roles in modulating the dynamics, organization, and function of membrane proteins [1-4]. A detailed understanding of the properties of lipid bilayers is central to biology, and can help the advancement of related research areas such as biomedicine and drug design [5-7]. Molecular dynamics (MD) simulations provide a powerful, complementary approach to experiments, and as such they have been used to study extensively many

Electronic supplementary material The online version of this article (https://doi.org/10.1007/s00894-019-3964-0) contains supplementary material, which is available to authorized users.

Mario Orsi

mario.orsi@uwe.ac.uk

1 Institute of Bioengineering, Queen Mary University of London, Mile End Road, London E1 4NS, UK

2 School of Engineering \& Materials Science, Queen Mary University of London, Mile End Road, London E1 4NS, UK

3 Department of Applied Sciences, University of the West of England, Coldharbour Lane, Bristol BS16 1QY, UK aspects of lipid bilayers [8-14]. Simulations are especially useful to study those properties that fluctuate substantially as a function of depth inside the bilayer, as experimental investigation is very arduous, mostly because of the small thickness $(\sim 45 \AA)$, high fluidity and heterogeneity of typical bilayers.

In this study, we use atomistic MD simulations to characterize a number of fundamental physical properties for various bilayers of different lipid compositions. In particular, we investigate multicomponent, asymmetric lipid bilayers with complex compositions mimicking mammalian, cancer, and bacterial plasma membranes.

The mammalian plasma membranes are typically made of approximately $65 \%$ glycerolipids, $10 \%$ sphingolipids, and $25 \%$ sterols, and have a highly asymmetric lipid composition [15-17]. Specifically, the extracellular leaflet is mostly composed of phosphatidylcholine (PC) lipids such as 1-palmitoyl-2-oleoyl-sn-glycero-3-phosphocholine (POPC), 1,2-dioleoyl-sn-glycero-3-phosphocholine (DOPC) and of sphingolipids such as sphingomyelin and glycosphingolipids. The intracellular leaflet on the other hand is enriched in phosphatidylethanolamine (PE) lipids such as 1palmatoyl-2-oleoyl-sn-glycero-3-phosphoethanolamine(POPE) and 1,2-dioleoyl-sn-glycero-3-phosphocholine (DOPE), and in negatively charged phosphatidylserine (PS) lipids such as 1-palmitoyl-2-oleoyl-sn-glycero-3-phospho-L-serine 
(POPS) and 1,2-dioleoyl-sn-glycero-3-phospho-L-serine (DOPS). While many phospholipids may show no preference to a specific leaflet of the membrane, PS lipids are known to be predominantly located in the inner leaflets of the cells [18-21]. A consequence of this irregular lipid composition is that the inner leaflet of the mammalian plasma membrane is anionic in nature $[15,16]$.

When healthy cells develop into cancer ones, the transformation is accompanied by a 5-9 times increase in PS lipids in the external leaflet; in fact, this change has been the primary mode of recognition of transformed cells by monocytes as part of the body's immune response [22, 23]. Indeed, in many studies, efforts have been made to exploit this expression of PS lipids in external leaflets to design cationic antimicrobial peptides (AMPs) and anticancer peptides (ACPs) that specifically target tumor cells, without harming the surrounding normal cells [24, 25]. Another alteration found in tumor cells is the reduction of cholesterol. Cholesterol is a major component of all eukaryotic membranes, where its presence induces increased ordering of lipid hydrocarbon chains [26-28], decreased lateral diffusion of lipids along the membrane plane [29] and increased lateral pressure profile magnitudes [30, 31].

In this study, we simulate both a mammalian and a cancer bilayer membrane models based on the representative compositions of Klahn et al. [32], which reflect the two aforementioned features of healthy mammalian cells and transformed cancer ones. It is important to stress that the models used in our work include only representative lipids of a limited number of prevalent species, and thus do not capture the full diversity of lipids in real cells; for example, predominant species such as polyunsaturated lipids, as well as PA, PI/PIPs and glycolipids, are not included. Especially considering the heterogeneity in saturation levels of lipid tails in real cells, the presence of lipids with only monounsaturated tails is a limitation of our study.

Regarding the bacterial membrane, we have approximated the complexity of real systems [33] with a model consisting of a 2:1 POPE:POPG lipid composition, which is arguably representative of the fundamental character of most Gram-negative bacterial membranes. In fact, the lipid composition used in our work has been used previously both in experiments [34] and MD simulations [35, 36]. In particular, the PG lipids induce a net negative charge on bacterial membranes (similarly to the effect of PS lipids on cancer membranes).

Apart from the three complex bilayers discussed above (mammalian, cancer, and bacterial), we also simulated homogeneous bilayers composed of individual lipids present in the complex systems, in order to investigate their contributions to the various properties analyzed. For each bilayer, we first calculate a number of basic membrane structural properties, such as area and volume per lipid, and bilayer thickness. We then place special focus on the transmembrane profiles of lateral pressure and dipole potential, as these fundamental properties, which vary significantly as a function of depth inside the membrane, are believed to play important roles in many membrane processes [37-39].

Considering the types of known interactions that exist at various depths inside the lipid membrane, an internal inhomogeneous distribution of lateral pressure is expected theoretically and can be predicted qualitatively [40]. Experiments have also been conducted to quantify the lateral pressure using pyrene moieties as probes to detect the local pressure change [41, 42]. However, only relative pressure changes in the tail regions could be obtained, and the accuracy of the experimental data is disputed [12, 41]. More recently, numerous molecular simulations have provided quantitative evidence that the lateral pressure profile is characterized by depth-dependent pressure variations of the order of hundreds of atmospheres [12, 40, 43]. Furthermore, it is believed that the corresponding forces affect embedded proteins and permeating molecules, with repercussions on many membrane phenomena $[1,39,44,45]$. For example, specific changes in the membrane lateral pressure may underpin the basic mechanism of general anesthesia by controlling the opening of ion channels that conduct neural signals [46]. An important factor that determines the shape and magnitude of the lateral pressure profile is the specific composition of the lipid bilayer. Notably, it has been shown that even small changes in the lipid composition can induce large variations in the pressure profiles [39]. Previous simulation studies have focused on the effects of cholesterol [30, 31], changing unsaturation levels of constituent lipids $[47,48]$ as well as the lamellar vs. nonlamellar lipid ratio using coarse-grained [49] and atomistic models [50]. Perrin et al. [51] have reported the pressure profiles for a 1:1 POPE:POPG, which is similar to our 2:1 POPE:POPG bacterial bilayer. However, as far as we are aware, no previous MD study has been reported on pressure profiles for the complex mammalian and cancer bilayer systems considered in this work.

The dipole potential is an intramembrane electrostatic potential which arises at the water-lipid interface due to the ordering of water molecular dipoles as well as dipolar charge distributions on lipid molecules [37, 52]. A direct measurement of the dipole potential would require the deployment of nanoscale electrodes at different depths within the bilayer, which has proved so far practically impossible [52]. It has been, however, possible to estimate the potential difference between the bilayer center and the outer water phase through indirect experimental measurements. In a pioneering study on ion transport [53], the transbilayer conductivity of two fat-soluble ions with similar sizes was observed to be drastically different, with the permeability of the negative ion $\left(\mathrm{TPB}^{-}\right)$being about $10^{5}$ times 
higher than the positive ion $\left(\mathrm{TPP}^{+}\right)$. This difference was ascribed to the existence of a positive electrostatic potential within the membrane. Some follow-up studies using varied methods and experiments confirmed the presence of such an electrostatic potential and estimated a magnitude of several hundreds of $\mathrm{mV}[52,54]$. It is crucial to note that the dipole potential fluctuates sharply within short distances of 2-3 nm, which is the approximate thickness of each of the monolayers in a typical bilayer. The corresponding electric field (which corresponds to the spatial derivative of the dipole potential) can therefore reach extremely large values, predicted to be in the range of $10^{7}-10^{9} \mathrm{~V} / \mathrm{m}$. As a result, many electrostatically driven phenomena, such as binding and permeation of charged or polar molecules, as well as dynamics and interactions of ligands and corresponding transmembrane proteins, may be affected by changes in dipole potential. Past studies have considered the effects of lipid composition on the dipole potential, with a focus on the headgroup type [55], ether or ester linkage type [56] and double bonds in the chain region [57]. An atomistic MD simulation study by Ding et al. [50] focused on the effects of altering the lamellar vs. nonlamellar lipid composition. Overall, previous studies have considered relatively simple systems, either homogeneous bilayers or comprising two lipid types. In this work, we report for the first time the dipole potential of more complex and biologically relevant bacterial, mammalian, and cancer model membranes.

\section{Methods}

\section{Lipid bilayer systems}

Details of the composition of the lipid bilayer systems simulated in this work are reported in Table 1.
In particular, we study four homogeneous systems, each containing a single lipid type: POPC, POPE, POPG, and POPS. All the PC, PE, PG and PS lipids in this work have 1phosphatidyl-2-oleoyl (PO) tails. The POPC system is then extended to include extra components, resulting in three mixed systems of incremental complexity, which include cholesterol (Chol) and palmitoylsphingomyelin (PSM): PC:Chol, PC:Chol:PSM and PC:Chol:PSM:PE. Palmitoylsphingomyelin (PSM) consists of 18:1-sphingosine and 16:0-palmitic acid. The molecular structures of all the lipid types simulated in this study are displayed in Fig. 1. Three additional systems are considered as models for bacterial, mammalian, and cancer membranes. Specifically, the bacterial membrane consists of a 2:1 PE:PG composition, following Horn et al. [58]. Regarding the mammalian and cancer membranes, their composition was taken from Klahn et al. [32]; supplementary Fig. S1 displays the percentage lipid composition and Table 2 reports their asymmetric lipid distribution in the outer and inner leaflets.

All systems were fully hydrated with water and were neutralized with counter-ions wherever necessary. The CHARMM36 force field and the corresponding TIP3P implementation were used to model the lipids and water, respectively $[59,60]$. System configurations were set up using Membrane Builder [61-63] from the Charmm-GUI [64]. In general, when constructing asymmetric bilayers, it is important to include the correct number of lipids in each leaflet, as any mismatch could introduce unrealistic effects, such as non-zero leaflet tension [65]. As a check on our systems, we carried out a number of extra simulations of symmetric bilayers, each comprising a lipid composition taken from each of the leaflets in the asymmetric systems. We then calculated the XY leaflet areas and compared them. As shown in the Supplementary Information (Fig. S3), after an initial equilibration the symmetric systems converge to
Table 1 Composition of the lipid bilayer systems simulated

\begin{tabular}{lllll}
\hline System & Lipid type $\left(\mathrm{N}_{\text {lipids }}\right)$ & $\mathrm{N}_{\text {water }}$ & $\mathrm{N}_{\text {ions }}$ & $\mathrm{N}_{\text {atoms }}$ \\
\hline POPC & PC (128) & 5760 & 0 & 33856 \\
POPE & PE (128) & 5760 & 0 & 33280 \\
POPG & PG (128) & 5760 & 128 & 33664 \\
POPS & PS (128) & 5760 & 128 & 33664 \\
PC:Chol & PC (134), Chol (66) & 9000 & 0 & 49840 \\
PC:Chol:PSM & PC (101), Chol (66), PSM (33) & 9000 & 0 & 49609 \\
PC:Chol:PSM:PE & PC (59), Chol (66), PSM (33), PE (42) & 9000 & 0 & 49231 \\
Bacterial & PE (88), PG (44) & 5940 & 44 & 34452 \\
Mammalian & PC (70), PE (83), PS (45), Chol (136), PSM (63) & 28000 & 45 & 127982 \\
Cancer & PC (79), PE (101), PS (86), Chol (68), PSM (66) & 28000 & 86 & 131633 \\
\hline
\end{tabular}

$\mathrm{N}_{\text {lipids }}$ is the total number of lipids, $\mathrm{N}_{\text {water }}$ is the total number of water molecules, $\mathrm{N}_{\text {ions }}$ is the total number of ions and $\mathrm{N}_{\text {atoms }}$ is the total number of atoms in the system. Lipid type names are abbreviated as follows: PC (POPC), PE (POPE), PG (POPG), PS (POPS), Chol (cholesterol) and PSM (palmitoylsphingomyelin) 


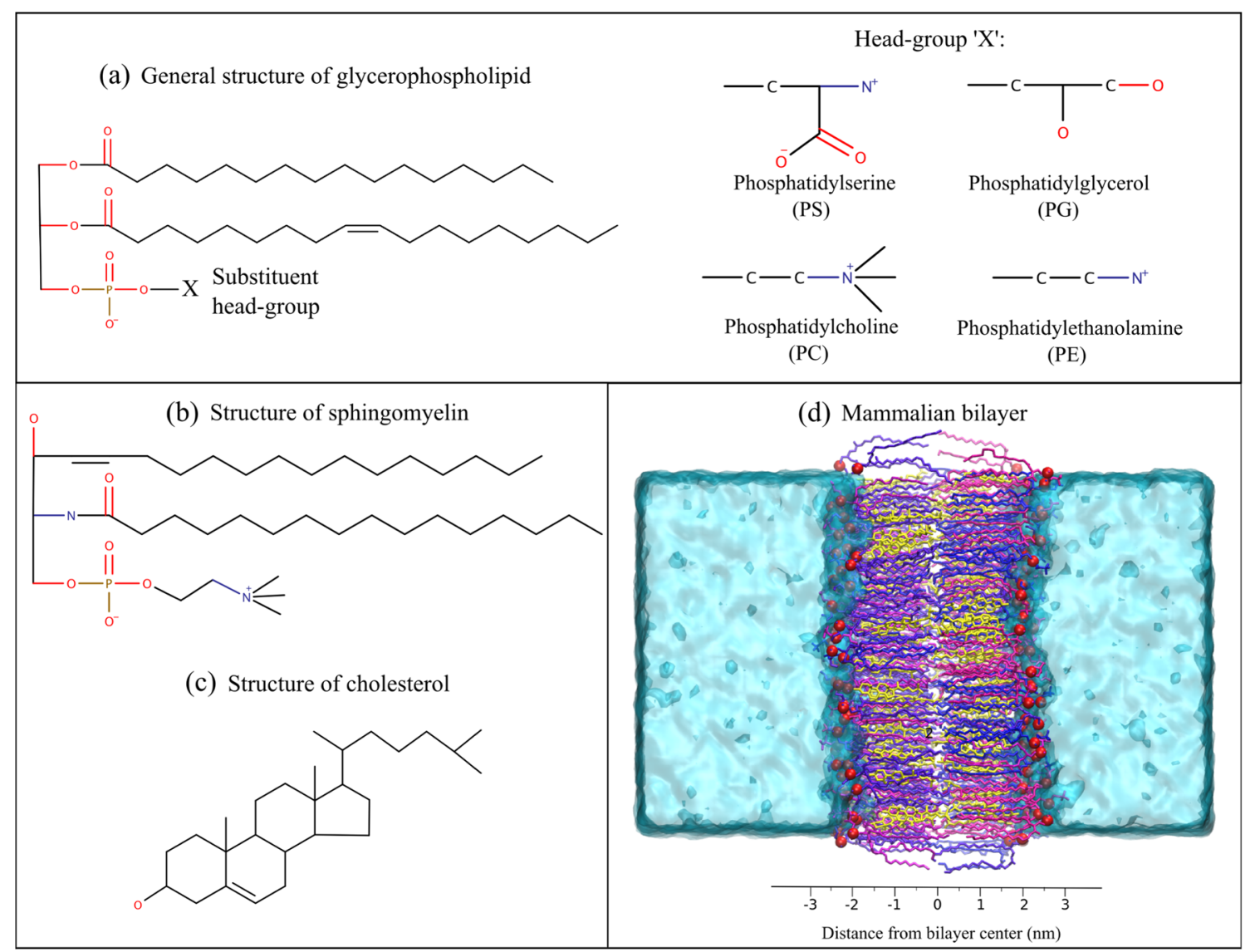

Fig. 1 Molecular structures of all a glycerophospholipids, b palmitoylsphingomyelin and $\mathbf{c}$ cholesterol used in this study. Explicit hydrogens are omitted for clarity. d A representative snapshot of the mammalian bilayer system (created in VMD [108]) simulated in this study, with an approximate scale of distance from the bilayer center. Different colors correspond to different lipid species. The lipid tails are displayed in licorice, phosphate atoms with VDW, and water molecules with QuickSurf representation

\section{Simulation details}

Molecular dynamics simulations were performed using Gromacs version $5.1[66,67]$. All the controls and bacterial systems were subjected to energy minimization and then NPT equilibration for $1 \mathrm{~ns}$. The systems were coupled to the velocity rescale thermostat [68] with a coupling time constant of $0.1 \mathrm{ps}$. Except for the POPE, all the other systems were simulated at $303 \mathrm{~K}$. The POPE bilayer was simulated at $310 \mathrm{~K}$ for consistency with the updated CHARMM36 force field [60]; such a slightly higher temperature typically used in previous simulations of the CHARMM36 model for POPE [60, 69] reproduces the correct liquid phase experimental behavior while preventing the formation of a gel phase (this is depicted in Fig. S2 in the Supplementary Information). Semi-isotropic pressure coupling [70-72] was carried out using the Berendsen barostat [73] for equilibration and the Parrinello-Rahman barostat [74] for production runs, with a coupling time constant of 2 ps. The SETTLE algorithm [75] was used to constrain bonds and angles in water molecules. All 
other hydrogen-related bonds were constrained using the LINCS algorithm [76], with two iterations in every step for correcting rotational effects and a numerical expansion up to the fourth order. Volume compressibility was chosen to be $4.5 \cdot 10^{-5}$ bar $^{-1}$. For short-range van der Waals and electrostatic cutoff, the default optimized distance of $1.2 \mathrm{~nm}$ was used and a force-switch potential modifier was applied from 1 to $1.2 \mathrm{~nm}$. Long-range electrostatic interactions were calculated using the particle mesh Ewald (PME) algorithm [77]. The neighbor list was updated using grid-based search procedure every five steps. Each system was simulated for 1 $\mu \mathrm{s}$; means and standard errors for the properties of interest were calculated using three trajectory blocks, corresponding to the intervals of 251-500, 501-750, and 751-1000 ns, while the initial $250 \mathrm{~ns}$ was regarded as equilibration.

Some extra steps were taken for equilibrating the mammalian, cancer, and the intermediate systems (PC:Chol:PSM and PC:Chol:PSM:PE) due to their complexity. In particular, these systems were subjected to stepwise energy minimization and equilibration under the NVT and NPT ensembles. The systems were coupled to the Berendsen thermostat at $303 \mathrm{~K}$ with a coupling time constant of 1 ps. The pressure was maintained at 1 atm using semi-isotropic pressure coupling with the Berendsen barostat [73]. For production runs, the thermostat and barostat were switched to velocity rescale and Parrinello-Rahman respectively, consistently with the other systems.

\section{Calculations and data analysis}

Structural properties Following a common convention, the lipid bilayers were set up along the $X Y$-plane, with the $Z$-axis thus crossing the membrane perpendicularly. The area per lipid $\left(A_{\mathrm{L}}\right)$ is defined as the cross-sectional area $\left(A_{\mathrm{XY}}\right)$ of the whole system along the bilayer surface plane ( $X Y$-plane), divided by half the total number of lipids $\left(N_{\mathrm{L}}\right)$ present in the bilayer, i.e., $A_{\mathrm{L}}=A_{\mathrm{XY}} /\left(N_{\mathrm{L}} / 2\right)$. An exception to this method, however, is the $\mathrm{PC}$ :Chol bilayer, for which the area per lipid for POPC in the presence of cholesterol was calculated using the freely available tool APL@VORO [78]. The volume per lipid $\left(V_{\mathrm{L}}\right)$ was calculated from the total volume of the simulation box ( $\left.V_{\text {box }}\right)$ as $V_{\mathrm{L}}=\left(V_{\text {box }}-V_{\text {water }}\right) / N_{\mathrm{L}}$, where $V_{\text {water }}$ is calculated from simulation of a pure water box at $303 \mathrm{~K}$ (at $310 \mathrm{~K}$ for POPE bilayer) and $1 \mathrm{~atm}$. The bilayer thickness $\left(d_{\mathrm{HH}}\right)$ was calculated as the head-to-head distance between the two peaks of the electron density profile. The electron density profiles were calculated using the gmx density tool in Gromacs, with the simulation box divided into 500 slabs parallel to the $X Y$-plane.

Lateral pressure profile To calculate the lateral pressure profile $\pi(z)$ we used GROMACS-LS [79-81], a custom version of GROMACS that calculates the local stress tensor in 3D. This package was used to rerun the simulated trajectories and output the local stress tensors. Since the long-range electrostatic solver is not implemented in GROMACS-LS, an increased cutoff distance of $2.0 \mathrm{~nm}$ was used for Coulomb interactions, as recommended by the package developers. The simulation box was divided into $0.1 \AA$ thick slabs parallel to the $X Y$-plane, with the lateral pressure $\pi\left(z_{\mathrm{s}}\right)$ at slab $s$ defined as:

$\pi\left(z_{\mathrm{s}}\right)=\frac{P_{\mathrm{xx}}\left(z_{\mathrm{s}}\right)-P_{\mathrm{yy}}\left(z_{\mathrm{s}}\right)}{2}-P_{\mathrm{zz}}\left(z_{\mathrm{s}}\right)$

where $P_{\mathrm{xx}}\left(z_{\mathrm{s}}\right), \quad P_{\mathrm{yy}}\left(z_{\mathrm{s}}\right)$ and $P_{\mathrm{zz}}\left(z_{\mathrm{s}}\right)$ are the diagonal elements of the pressure tensor for slab $s$, along the $X, Y$ and $Z$ axes respectively. All the profiles have been smoothed by adaptive high-order spline fitting for clarity [82].

Dipole potential We calculated the transmembrane dipole potential $\psi(z)$ using the Gromacs tool gmx potential. In particular, the potential is obtained by summing the charge density distribution $\rho(z)$ per slab, where the simulation box was divided into 500 slabs of $\sim 0.18 \AA$ thickness each. The dipole potential is obtained by double integration using the following expression [71]:

$\psi(z)=-\frac{1}{\epsilon_{0}} \int_{0}^{z} \int_{0}^{z^{\prime}} \rho\left(z^{\prime \prime}\right) d z^{\prime \prime} d z^{\prime}$

where $\epsilon_{0}$ is the electrostatic permittivity in vacuum.

\section{Results and discussion}

\section{Structural properties}

Simulation results for area per lipid $A_{\mathrm{L}}$, volume per lipid $V_{\mathrm{L}}$, and bilayer thickness $d_{\mathrm{HH}}$ are reported in Table 3, together with available literature data from experiments and previous atomistic simulations.

For the homogeneous bilayers, as well as the PC:Chol system, our results are in good agreement with the values previously reported in the literature, thus validating our simulation setup.

The bacterial bilayer, being composed of POPE and POPG lipids in the ratio 2:1, exhibits intermediate values for its structural properties, although closer to the homogeneous POPE values. This is expected, due to POPE's higher concentration in the bacterial bilayer, however it appears that POPE's contribution is amplified in relation to its concentration. In particular, the bacterial bilayer exhibits condensation, in that the lipid area for the two-lipid mixture is lower than expected by simply taking a weighted average over the individual lipid areas of the POPE and POPG bilayers. This effect is also reflected in the electron density 
Table 3 Structural properties

\begin{tabular}{|c|c|c|c|c|c|}
\hline \multicolumn{3}{|l|}{ This Work } & \multicolumn{3}{|l|}{ Previous studies } \\
\hline$A_{L}\left(\AA^{2}\right)$ & $\mathrm{V}_{\mathrm{L}}\left(\AA^{3}\right)$ & $\mathrm{d}_{\mathrm{HH}}(\AA)$ & $A_{L}\left(\AA^{2}\right)$ & $\mathrm{V}_{\mathrm{L}}\left(\AA^{3}\right)$ & $\mathrm{d}_{\mathrm{HH}}(\AA)$ \\
\hline \multicolumn{6}{|l|}{ POPC } \\
\hline \multirow[t]{2}{*}{$63.1(0.1)$} & $1231.2(0.1)$ & $37.5(0.3)$ & $64.3^{\mathrm{E}},[93]$ & $1256^{\mathrm{E},}[94]$ & $37^{\mathrm{E}},[94]$ \\
\hline & & & $64.7^{\mathrm{S},}[60]$ & $1191.9^{\mathrm{S},}[95]$ & $38^{\mathrm{S}},[95]$ \\
\hline \multicolumn{6}{|l|}{$\mathrm{POPE}_{310 \mathrm{~K}}$} \\
\hline \multirow[t]{3}{*}{$55.6(0.1)$} & $1176.8(0.1)$ & $43.4(0.2)$ & $56.6^{\mathrm{E},}[96]$ & $1180_{308 \mathrm{~K}} \mathrm{E},[97]$ & $39.5_{308 \mathrm{~K}^{\mathrm{E}},[97]}$ \\
\hline & & & $59.2_{310 \mathrm{~K}} \mathrm{~S},[60]$ & $1134.9_{310 \mathrm{~K}} \mathrm{~S},[95]$ & $41_{310 K} \mathrm{~S},[95]$ \\
\hline & & & $58.8_{310 \mathrm{~K}} \mathrm{~S},[69]$ & & \\
\hline \multicolumn{6}{|l|}{ POPG } \\
\hline \multirow[t]{3}{*}{$64.6(0.1)$} & $1226.6(0.1)$ & $38.5(0.2)$ & $66^{\mathrm{E} / \mathrm{S}},[98]$ & $1203^{\mathrm{E} / \mathrm{S}},[98]$ & $36.7^{\mathrm{E} / \mathrm{S}},[98]$ \\
\hline & & & $66.3_{310 K} \mathrm{~S},[99]$ & 1208.7 [100] & $36.3_{310 \mathrm{~K}} \mathrm{~S},[99]$ \\
\hline & & & $67.2^{\mathrm{S},}[101]$ & $1151^{\mathrm{S},}[101]$ & $36.3^{\mathrm{S},}[101]$ \\
\hline \multicolumn{6}{|l|}{ POPS } \\
\hline \multirow[t]{3}{*}{$55.8(0.3)$} & $1196.01(0.3)$ & $43.2(0.5)$ & $62.7_{298 \mathrm{~K}}{ }^{\mathrm{E} / \mathrm{S},}[102]$ & $1198.5_{308 K^{E}}{ }^{E},[102]$ & $42.2308 \mathrm{~K}^{\mathrm{E},}[102]$ \\
\hline & & & $55_{300 K} \mathrm{~S},[103]$ & $1194_{300 K} \mathrm{~S},[103]$ & $40.6_{298 K} \mathrm{~S},[102]$ \\
\hline & & & $57.5^{\mathrm{S},}[101]$ & $1120.5^{\mathrm{S},}[101]$ & $42.4^{\mathrm{S},}[101]$ \\
\hline \multicolumn{6}{|l|}{ PC:Chol } \\
\hline \multirow[t]{2}{*}{$49.45^{*}(0.1)$} & $1027.5(0.1)$ & $45.4(0.1)$ & $45.1_{321 \mathrm{~K}} \mathrm{E}, 50 \%,[85]$ & not available & $44.5_{300 \mathrm{~K}} \mathrm{~S}, 30 \%,[104]$ \\
\hline & & & $51.1_{298 \mathrm{~K}}^{\mathrm{S}, 40 \%,}[105]$ & & \\
\hline \multicolumn{6}{|l|}{ Bacterial } \\
\hline \multirow[t]{2}{*}{$56.8(0.1)$} & $1186.6(0.1)$ & $40.8(0.1)$ & $58.3^{\mathrm{S}, 3: 1,}[106]$ & not available & not available \\
\hline & & & $61.5_{310 \mathrm{~K}} \mathrm{~S}, 3: 1,[107]$ & & \\
\hline \multicolumn{6}{|l|}{ Mammalian } \\
\hline $42.1(0.1)$ & $989.5(0.3)$ & $47.3(0.2)$ & not available & not available & not available \\
\hline \multicolumn{6}{|l|}{ Cancer } \\
\hline $46.1(0.1)$ & $1085.5(0.3)$ & $45.9(0.4)$ & not available & not available & not available \\
\hline
\end{tabular}

$\mathrm{A}_{\mathrm{L}}, \mathrm{V}_{\mathrm{L}}$ and $\mathrm{d}_{\mathrm{HH}}$ represent the area per lipid, volume per lipid and bilayer thickness, respectively. Standard errors are reported in round brackets. Superscripts 'E' and 'S' correspond to values derived from experiments and simulations, respectively; 'E/S' indicates values derived from a combined experimental and simulation approach. The temperature is $303 \mathrm{~K}$ unless otherwise stated as subscript. For PC:Chol bilayer and bacterial bilayers, the relevant cholesterol concentration and PE:PG lipid ratios are reported as superscripts. Last superscript numbers are references. ${ }^{*}$ The value reported for area per lipid for PC:Chol is of POPC only

profiles, reported in Fig. 2. Specifically, it can be seen that the bacterial profile exhibits peaks that are larger than the constituent lipid profiles.

The mammalian lipid bilayer, which is a complex mixture of five different types of lipids, exhibits the lowest area and volume per lipid $\left(\sim 42 \AA^{2}\right.$ and $\sim 989 \AA^{3}$, respectively) and the highest bilayer thickness of $\sim 47 \AA$. This can be attributed primarily to the condensation effect induced by the predominance of cholesterol (34\%) in the bilayer. A number of studies have indeed demonstrated that the presence of cholesterol induces a reduction in $A_{\mathrm{L}}$ and $V_{\mathrm{L}}$ and an increase in $d_{\mathrm{HH}}$ in phospholipid bilayers [83-87]. Our own PC:Chol bilayer $\left(A_{\mathrm{L}}: \sim 45 \AA^{2}\right)$, with one-third cholesterol content shows a $\sim 30 \%$ reduction in $A_{\mathrm{L}}$ as compared to POPC $\left(\sim 63 \AA^{2}\right)$, in close agreement with such previous studies [83-87]. Compared to the mammalian bilayer, the cancer composition is characterized by a decrease in cholesterol and an increase in PS and PE lipids (also see supplementary Fig. S1). Such changes bring about an $\sim 18 \%$ increase in $A_{\mathrm{L}}$, from $\sim 42 \AA^{2}$ to $\sim 46 \AA^{2}$, an $\sim 10 \%$ increase in $V_{\mathrm{L}}$, from $\sim 990 \AA^{3}$ to $\sim 1085 \AA^{3}$, and a $\sim 3 \%$ decrease in bilayer thickness, from $\sim 47.3 \AA$ to $\sim 45.9 \AA$. The presence of cholesterol in lipid bilayers is known to increase the ordering of lipid chains by intercalating in free spaces between them [88], effectively decreasing the lipid area; since a lipid membrane 


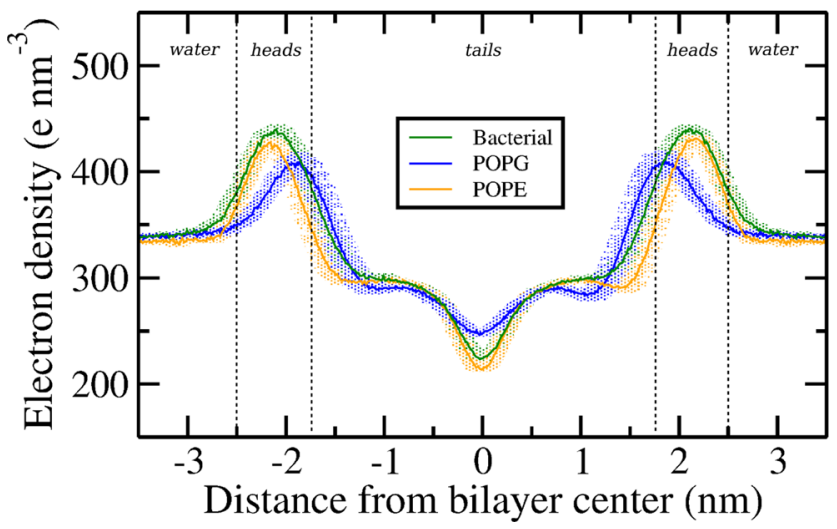

Fig. 2 Electron densities of bacterial, POPE, and POPG bilayers. Shaded areas represent error bars. The vertical dotted lines represent approximate boundaries for the regions occupied primarily by water, lipid heads, and tails

behaves as an incompressible fluid [86], this decrease in the area per lipid causes the bilayer thickness to increase. The difference in lipid composition between mammalian and cancer bilayers is also reflected in the electron densities, as illustrated in Fig. 3. It can be noted, in particular, that the mammalian profile shows a broadening of its peaks in the headgroup region compared to its cancer counterpart, without any significant change in the magnitudes.

\section{Lateral pressure profile}

The lateral pressure profiles for all the systems simulated in this study are shown in Figs. 4 and 5. Specifically, Fig. 4 shows profiles for the bacterial lipid bilayer, together with the constituent POPE and POPG homogeneous systems. All three pressure profiles display a similar general trend. In particular, corresponding to the profile extremes at the outer aqueous phase, the lateral pressure is close to zero,

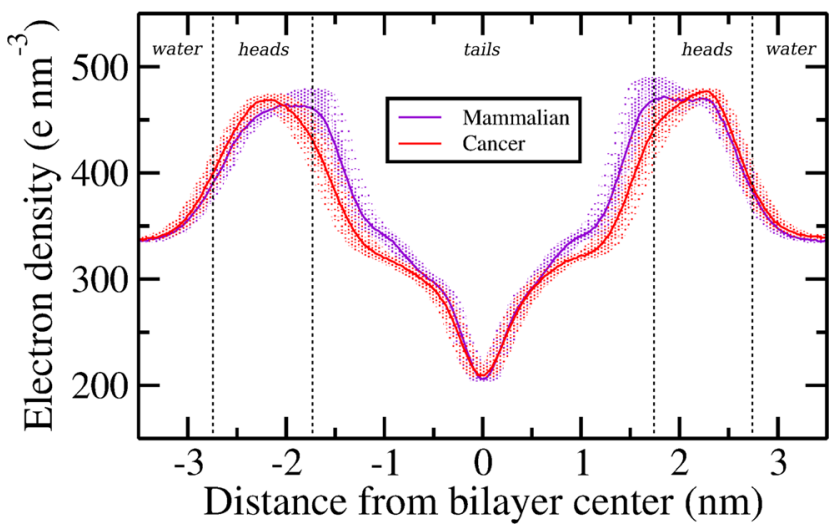

Fig. 3 Electron densities of mammalian and cancer bilayers. Shaded areas represent error bars. The vertical dotted lines represent approximate boundaries for the regions occupied primarily by water, lipid heads, and tails

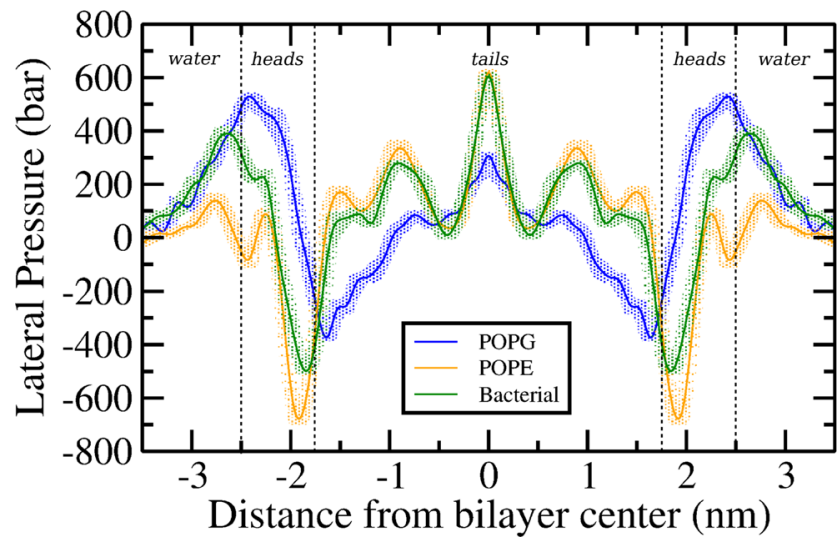

Fig. 4 Lateral pressure profiles for the bacterial, POPE, and POPG bilayers. Shaded areas represent error bars. The vertical dotted lines represent approximate boundaries for the regions occupied primarily by water, lipid heads, and tails

as expected. Upon approaching the membrane, the lateral pressure rises and forms a positive peak at the interface between the water and lipid heads regions. Positive lateral pressure values indicate repulsive forces, acting to increase the area of the bilayer. Proceeding deeper into the bilayer, the lateral pressure declines sharply, forming deep troughs near the interface between the lipid heads and tails regions. Negative lateral pressure reflects the presence of attractive forces that act to reduce the bilayer area. In fact, the pressure troughs are a manifestation of the hydrophobic effect, whereby attractive lateral forces minimize the contact area between the hydrophobic core of the bilayer (comprising the hydrocarbon tails) and the hydrophilic environment (comprising lipid headgroups and water).

In the center of the bilayer, corresponding to the core of hydrophobic tails, the POPE and bacterial pressure profiles have five positive peaks, with four corresponding local minima in between them. POPG instead features a single central maximum. In general, positive pressure peaks in the lipid tails region represent repulsive forces arising from entropy loss [39]. Specifically, the tight molecular packing in the bilayer core forces the lipid tails to stretch, therefore losing entropy when compared to the isolated "free" tails, ultimately causing significant intermolecular repulsion in the bilayer center $[89,90]$.

While all three profiles in Fig. 4 display similar qualitative features, important differences can be observed in relation to the different lipid composition. At the interface between the water and lipid heads regions, at a distance of approximately $\sim 2.5 \mathrm{~nm}$ from the bilayer center, the POPG system displays a maximum positive pressure of $\sim 530$ bar, which is substantially larger than the value for the corresponding peaks in the bacterial (PE:PG) and POPE bilayers. Such large magnitude of the positive 


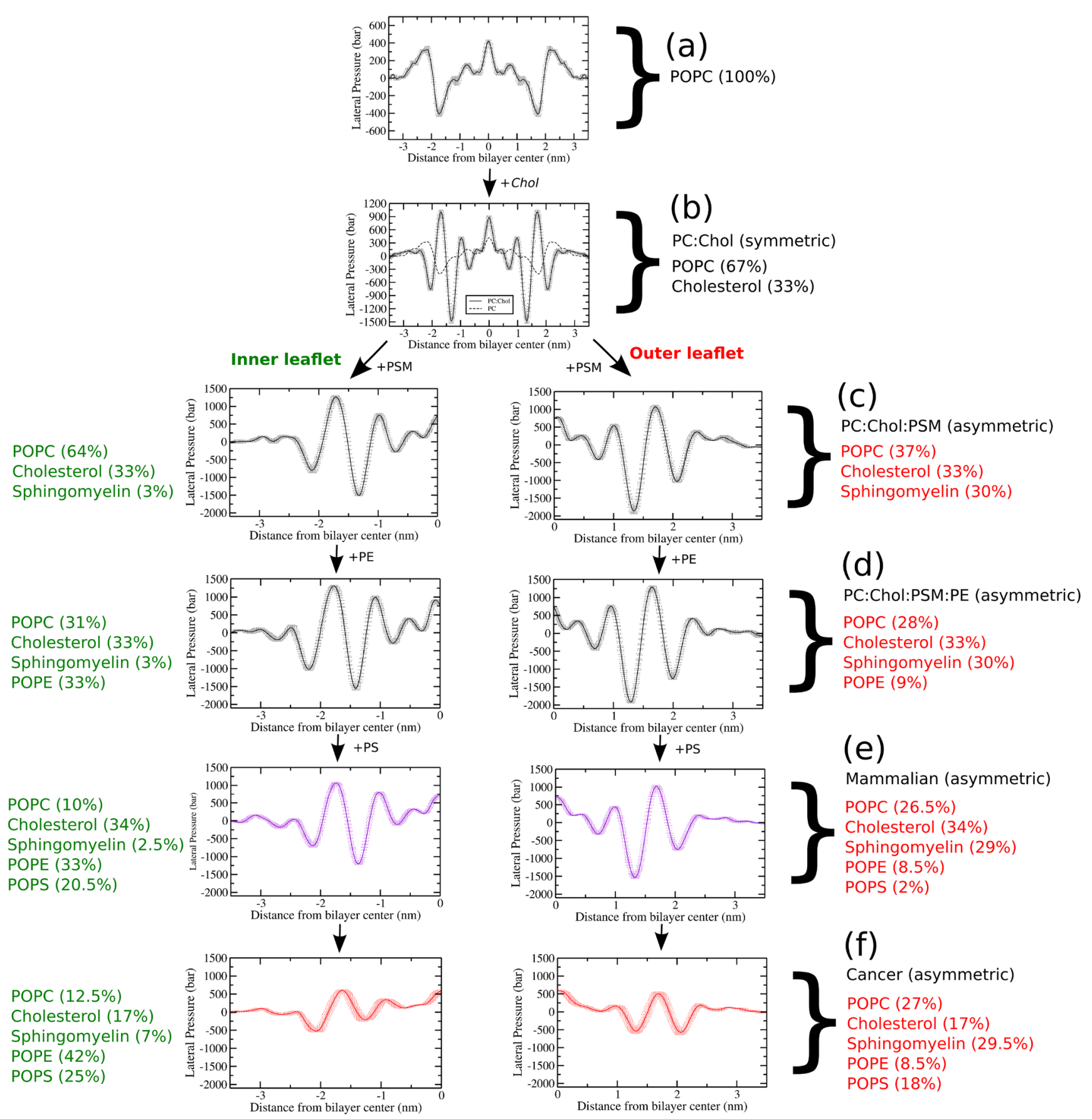

Fig. 5 Lateral pressure profiles of different bilayer systems with varying compositions. Shaded areas represent error bars. The systems vary in complexity starting from the top, from pure, symmetric POPC a and PC:Chol $\mathbf{b}$ bilayers to the mammalian $\mathbf{e}$ and cancer $\mathbf{f}$ bilayers at

peak for POPG can be attributed to significant headhead electrostatic repulsion, a consequence of POPG's net charge; in fact, we observed similarly high repulsion for a homogeneous bilayer comprising POPS, which also carries a net charge (see Fig. S4 in the Supplementary Information). Overall, in the water and heads regions, the bacterial bilayer displays a peak pressure of $\sim 400$ bar, a value intermediate the bottom. Pressure profiles from asymmetric bilayers $\mathbf{c}-\mathbf{f}$ are plotted separately, with the ones on the right from the outer leaflet and the ones on the left from the inner leaflet

between the large POPG peak of $\sim 530$ bar and the lower value of $\sim 140$ bar for the pure POPE bilayer. The POPE pressure profile is also characterized by the presence of a small trough in the heads region at about $\sim 2.4 \mathrm{~nm}$ distance from the bilayer center, as previously observed for a DOPE bilayer [50]. Regarding the pressure troughs near the headstails interface, the POPE system features the most negative 
value of $\sim-720 \mathrm{bar}$, while the bacterial and POPG bilayers feature pressures of $\sim-550$ and $\sim-400$ bar, respectively. In the tails region, the POPE and bacterial bilayers show large positive peaks in the range of $\sim 300$ to $\sim 400$ bar.

Regarding the mammalian and cancer bilayers, we have attempted to isolate the different contributions to the lateral pressure profiles from various lipid types. In particular, starting from the pure POPC bilayer, we have progressively added the required lipid species and monitored the corresponding effects on the pressure profile, until the attainment of the complete mammalian and cancer bilayer compositions. The results obtained for the various systems simulated are reported in Fig. 5. Starting from the top, the POPC bilayer (Fig. 5a) shows a pressure profile qualitatively similar to the previously discussed POPG bilayer, with large positive peaks in the heads region and a few small positive peaks in the tails region. The profile changes its structure and magnitude significantly with the addition of cholesterol (Fig. 5b); in particular, more peaks and troughs emerge, in agreement with previous studies [30, 31].

Noting that the cholesterol content in the mammalian bilayer is $34 \%$ of all lipids, we maintain constant cholesterol levels in both the upper and lower leaflets, while adding different lipids in different concentrations in the subsequent bilayers to make up the final lipid composition in the mammalian and cancer bilayers. The replacement of a proportion of POPC lipids with PSM (Fig. 5c) and PE (Fig. 5d) in the subsequent bilayers marginally increases the magnitudes of the peaks and troughs in the PC:Chol:PSM and PC:Chol:PSM:PE bilayers. The subsequent addition of PS lipids, which completes the formation of the mammalian bilayer, induces a small but noticeable reduction in the peak magnitudes (Fig. 5e). It can be observed that there is high similarity between the pressure profiles of PC:Chol and the more complex mammalian bilayers, indicating that cholesterol has a predominant role in shaping the lateral pressure profile. The PC:Chol and mammalian profiles are superimposed in Fig. S5 in the Supplementary Information. While the pressure profiles of the two bilayers are very similar in the outer leaflet, the mammalian pressure profile in the inner leaflet exhibits much higher magnitudes (up to $\sim 800$ bar and $\sim 1070$ bar at a distance of $1 \mathrm{~nm}$ and $1.75 \mathrm{~nm}$ from the bilayer center, respectively). The inner leaflet pressure profile of the mammalian bilayer also shows an outward shift in the lipid tails region, along with the appearance of a local trough in the lipid heads region $(\sim-$ 175 bar at a distance of $\sim 2.7 \mathrm{~nm}$ from the bilayer center). This behavior could be attributed to a higher concentration of PE lipids (33\%) in the inner leaflet of the mammalian bilayer, in qualitative agreement with our previous study on mixed DOPC/DOPE bilayers [50]. Compared to the mammalian system, the cancer bilayer (Fig. 5f) features greatly reduced peak magnitudes, which can be ascribed to the halving of its cholesterol content.

\section{Dipole potential}

The dipole potential profiles $\psi(z)$ for POPG, POPE, and the mixed PE:PG bacterial systems are displayed in Fig. 6, while the profiles for the cancer and mammalian bilayers are displayed in Fig. 7. While all profiles share similar qualitative characteristics, there are significant composition-dependent differences in the magnitude of the potentials.

Figure 6 shows that, starting from the initial zero reference value in the aqueous phase, the dipole potential rises rapidly across the lipid heads region, up to peak values of $\sim 370 \mathrm{mV}$ for POPG, $\sim 530 \mathrm{mV}$ for the bacterial bilayer and $\sim 640 \mathrm{mV}$ for POPE, at a distance of $\sim 1.3$ $\mathrm{nm}$ from the bilayer center. The profiles then form a local minimum at $\sim 0.8 \mathrm{~nm}$ from the bilayer center, at a depth roughly corresponding to the presence of the lipid tails unsaturation. The profiles then reach a global maximum in the bilayer center, with values of $\sim 500 \mathrm{mV}$ for POPG, $\sim 750$ $\mathrm{mV}$ for the bacterial bilayer and $\sim 900 \mathrm{mV}$ for the POPE bilayer. These central peaks are associated with the presence of partial charges, and corresponding dipoles, at the tails methyl termini. Our results for POPE are consistent with the previously reported atomistic simulation results of pure DOPC, DOPE, and mixed DOPC-DOPE bilayers [50, 70, 91] while for the POPG bilayers, our profile agrees with previous results by Zhao et al. [92].

Figure 7 reports the dipole potential profiles for the mammalian and cancer bilayers. Starting from the zero reference value in the water phase, the potential profiles rise towards the heads region up to peak values of $\sim 540 \mathrm{mV}$ for the mammalian and $\sim 560 \mathrm{mV}$ for the cancer system. Proceeding towards the bilayer center, a noticeable local

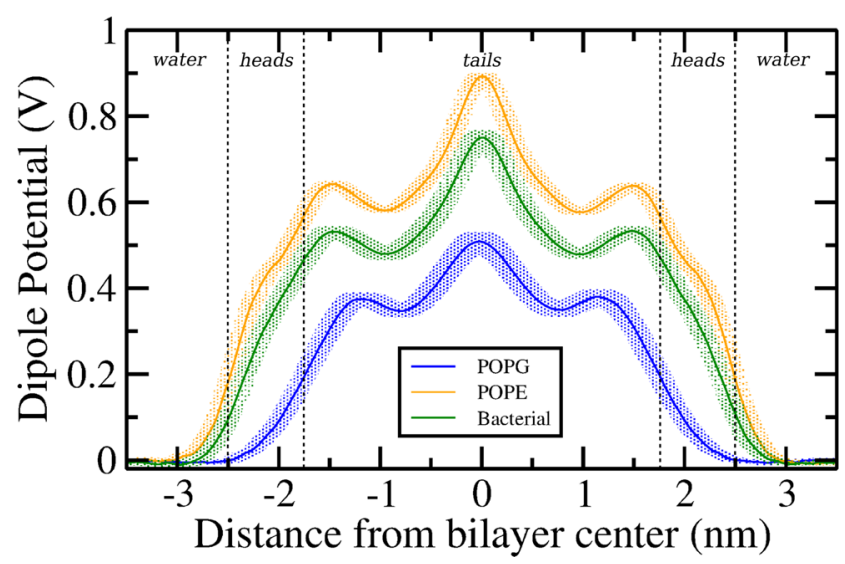

Fig. 6 Dipole potential profiles for bacterial, POPE, and POPG bilayers. Shaded areas represent error bars. The vertical dotted lines represent approximate boundaries for the regions occupied primarily by water, lipid heads, and tails 


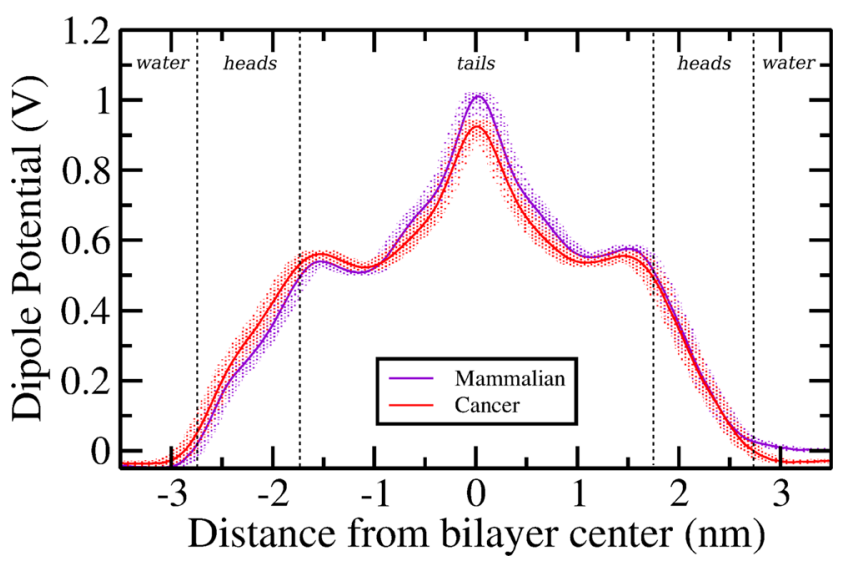

Fig. 7 Dipole potential profiles for cancer and mammalian bilayers. Shaded areas represent error bars. The vertical dotted lines represent approximate boundaries for the regions occupied primarily by water, lipid heads, and tails

minimum is present (although less prominent compared to the bacterial lipid bilayer results considered previously). The profiles then form a global maximum in the bilayer center, with a peak value of $\sim 985 \mathrm{mV}$ for the mammalian and $\sim 920 \mathrm{mV}$ for the cancer system. Upon entering the internal leaflet (corresponding to positive distances from the bilayer center in our convention), the profiles drop towards the secondary peaks near the heads-tails interface region, with values of $\sim 555 \mathrm{mV}$ for the mammalian and $\sim 525 \mathrm{mV}$ for the cancer system. Overall, these results show that the dipole potential is not significantly sensitive to changes in cholesterol and PS lipids.

\section{Conclusions}

In this work, we have used atomistic molecular dynamics simulations to study structural and depth-dependent properties of complex lipid bilayers with biologically relevant lipid compositions. In particular, we analyzed key properties of a model bacterial bilayer in terms of the contributions from the constituent lipid types. With regards to the lateral pressure profile, we found that the 2:1 POPE:POPG bacterial bilayer profile is mostly shaped by the POPE contribution in the central tails region, characterized by prominent pressure peaks of high magnitude, while the POPG contribution is predominant in the water and heads region. Regarding the dipole potential, the profiles of the bacterial system and those of the constituent lipids share the same qualitative features, while the magnitudes reflect the different composition. In particular, the bacterial profile, while bounded by the POPE and POPG bilayer profiles, has magnitudes closer to those of the POPE bilayer, consistently with the higher POPE concentration, compared to POPG, in the bacterial system. We also focused on the effects of changes in the lipid composition between mammalian and cancer bilayers. Cholesterol was identified as the predominant contributor to the lateral pressure in both complex bilayers. Specifically, the peak pressure magnitudes in the cancer profile were observed to be sharply reduced compared to those in the mammalian profile, corresponding to a $50 \%$ decrease in cholesterol in the cancer bilayer compared to the mammalian one. However, regarding the dipole potential, no noticeable differences were observed between the mammalian and cancer systems. To our knowledge, this is the first report of transmembrane lateral pressure and dipole potential profiles for model bacterial, mammalian, and cancer lipid bilayers.

Acknowledgments This project made use of time on the ARCHER supercomputer via the UK High-End Computing Consortium for Biomolecular Simulation (HECBioSim), supported by EPSRC (grant no. EP/L000253/1). GS acknowledges financial support from the Institute of Bioengineering at Queen Mary University of London.

Open Access This article is distributed under the terms of the Creative Commons Attribution 4.0 International License (http:// creativecommons.org/licenses/by/4.0/), which permits unrestricted use, distribution, and reproduction in any medium, provided you give appropriate credit to the original author(s) and the source, provide a link to the Creative Commons license, and indicate if changes were made.

Publisher's note Springer Nature remains neutral with regard to jurisdictional claims in published maps and institutional affiliations.

\section{References}

1. Mouritsen OG (2005) Life - as a matter of fat. The emerging science of lipidomics, 1st edn. Springer, Berlin

2. Alberts B, Johnson A, Lewis J, Raff M, Roberts K, Walter P (2002) Molecular biology of the cell, 4 th edn. Garland Science

3. Smith AW (2012) Lipid-protein interactions in biological membranes: a dynamic perspective. Biochimica et Biophysica Acta (BBA) - Biomembranes 1818(2):172-177

4. Spector AA, Yorek MA (1985) Membrane lipid composition and cellular function. J Lipid Res 26(9):1015-1035

5. Prausnitz MR, Langer R (2008) Transdermal drug delivery. Nat Biotechnol 26(11):1261-1268

6. Orsi M, Essex JW (2010) Permeability of drugs and hormones through a lipid bilayer: insights from dual-resolution molecular dynamics. Soft Matter 6(16):3797

7. Martin LJ, Chao R, Corry B (2014) Molecular dynamics simulation of the partitioning of benzocaine and phenytoin into a lipid bilayer. Biophys Chem 185:98-107

8. Lyubartsev AP, Rabinovich AL (2011) Recent development in computer simulations of lipid bilayers. Soft Matter 7(1):25-39

9. Orsi M, Sanderson W, Essex JW (2007) Coarse-grain modelling of lipid bilayers: a literature review. In: Hicks MG, Kettner $\mathrm{C}$ (eds) Molecular interactions - bringing chemistry to life. Beilstein-Institut, Frankfurt, pp 185-205

10. Tieleman DP, Marrink SJ, Berendsen HJC (1997) A computer perspective of membranes: molecular dynamics studies of lipid bilayer systems. Biochimica et Biophysica Acta (BBA) Reviews on Biomembranes 1331(3):235-270 
11. Piggot TJ, Piñeiro Á, Khalid S (2012) Molecular dynamics simulations of phosphatidylcholine membranes: a comparative force field study. J Chem Theory Comput 8(11):4593-4609

12. Samuli Ollila OH, Vattulainen I (2010) Chapter 2 lateral pressure profiles in lipid membranes: dependence on molecular composition. In: Molecular simulations and biomembranes: from biophysics to function. The Royal Society of Chemistry, pp 26-55

13. Siani P, de Souza RM, Dias LG, Itri R, Khandelia H (2016) An overview of molecular dynamics simulations of oxidized lipid systems, with a comparison of ELBA and MARTINI force fields for coarse-grained lipid simulations. Biochimica et Biophysica Acta (BBA) - Biomembranes 1858(10):2498-2511

14. Grasso G, Muscat S, Rebella M, Morbiducci U, Audenino A, Danani A, Deriu MA (2018) Cell penetrating peptide modulation of membrane biomechanics by molecular dynamics. J Biomech 73:137-144

15. van Meer G, Voelker DR, Feigenson GW (2008) Membrane lipids: where they are and how they behave. Nat Rev Mol Cell Biol 9(2):112-124

16. van Meer G, de Kroon AIPM (2011) Lipid map of the mammalian cell. J Cell Sci 124(Pt 1):5-8

17. Shevchenko A, Simons K (2010) Lipidomics: coming to grips with lipid diversity. Nat Rev Mol Cell Biol 11(8):593-598

18. Op den Kamp JAF (1979) Lipid asymmetry in membranes. Annu Rev Biochem 48(1):47-71

19. Gordesky SE, Marinetti GV (1973) The asymmetric arrangement of phospholipids in the human erythrocyte membrane. Biochem Biophys Res Commun 50(4):1027-1031

20. Verkleij AJ, Zwaal RF, Roelofsen B, Comfurius P, Kastelijn D, van Deenen LL (1973) The asymmetric distribution of phospholipids in the human red cell membrane. A combined study using phospholipases and freeze-etch electron microscopy. Biochimica et Biophysica Acta (BBA) - Biomembranes 323(2): 178-193

21. Rothman JE, Lenard J (1977) Membrane asymmetry. Science 195(4280):743-753

22. Utsugi T, Schroit AJ, Connor J, Bucana CD, Fidler IJ (1991) Elevated expression of phosphatidylserine in the outer membrane leaflet of human tumor cells and recognition by activated human blood monocytes. Cancer Res 51(11):3062-3066

23. Connor J, Bucana C, Fidler IJ, Schroit AJ (1989) Differentiationdependent expression of phosphatidylserine in mammalian plasma membranes: quantitative assessment of outer-leaflet lipid by prothrombinase complex formation. Proc Natl Acad Sci U S A 86(9):3184-3188

24. Iwasaki T, Ishibashi J, Tanaka H, Sato M, Ai A, Taylor D, Yamakawa M (2009) Selective cancer cell cytotoxicity of enantiomeric 9-mer peptides derived from beetle defensins depends on negatively charged phosphatidylserine on the cell surface. Peptides 30(4):660-668

25. Hoskin DW, Ramamoorthy A (2008) Studies on anticancer activities of antimicrobial peptides. Biochimica et Biophysica Acta (BBA) - Biomembranes 1778(2):357-375

26. Oldfield E, Meadows M, Rice D, Jacobs R (1978) Spectroscopic studies of specifically deuterium labeled membrane systems. Nuclear magnetic resonance investigation of the effects of cholesterol in model systems. Biochemistry 17(14):2727-2740

27. Urbina JA, Pekerar S, Le HB, Patterson J, Montez B, Oldfield E (1995) Molecular order and dynamics of phosphatidylcholine bilayer membranes in the presence of cholesterol, ergosterol and lanosterol: a comparative study using $2 \mathrm{~h}-$, 13C- and 31PNMR spectroscopy. Biochimica et Biophysica Acta (BBA) Biomembranes 1238(2):163-176
28. Thalia T, Mills G, Toombes ES, Tristram-Nagle S, Smilgies DM, Feigenson GW, Nagle JF (2008) Order parameters and areas in fluid-phase oriented lipid membranes using wide angle X-ray scattering. Biophys J 95(2):669-681

29. Filippov A, Orädd G, Lindblom G (2003) The effect of cholesterol on the lateral diffusion of phospholipids in oriented bilayers. Biophys J 84(5):3079-3086

30. Patra M (2005) Lateral pressure profiles in cholesterol-DPPC bilayers. Eur Biophys J 35(1):79-88

31. Ollila OHS, Rog T, Karttunen M, Vattulainen I (2007) Role of sterol type on lateral pressure profiles of lipid membranes affecting membrane protein functionality: comparison between cholesterol, desmosterol, 7-dehydrocholesterol and ketosterol. J Struct Biol 159(2 SPEC. ISS.):311-323

32. Klähn M, Zacharias M (2013) Transformations in plasma membranes of cancerous cells and resulting consequences for cation insertion studied with molecular dynamics. Phys Chem Chem Phys: PCCP 15(34):14427-41

33. Sohlenkamp C, Geiger O (2015) Bacterial membrane lipids: diversity in structures and pathways. FEMS Microbiol Rev 1:133-159

34. Merz C, Knoll W, Textor M, Reimhult E (2008) Formation of supported bacterial lipid membrane mimics. Biointerphases 3(2):41-50

35. Horn JN, Sengillo JD, Lin D, Romo TD, Grossfield A (2012) Characterization of a potent antimicrobial lipopeptide via coarsegrained molecular dynamics. Biochim Biophys Acta Biomembr 1818(2):212-218

36. Horn JN, Romo TD, Grossfield A (2013) Simulating the mechanism of antimicrobial lipopeptides with all-atom molecular dynamics. Biochemistry 52(33):5604-5610

37. Wang L (2012) Measurements and implications of the membrane dipole potential. Annu Rev Biochem 81:615-635

38. Cantor RS (1999) Lipid composition and the lateral pressure profile in bilayers. Biophys J 76(5):2625-2639

39. Cantor RS (1997) Lateral pressure in cell membranes: a mechanism for modulation of protein function. J Phys Chem 101(96):1723-1725

40. Cevc G, Marsh D (1987) Phospholipid bilayers: physical principles and models. Cell biology. Wiley

41. Templer RH, Castle SJ, Curran AR, Rumbles G, Klug DR (1998) Sensing isothermal changes in the lateral pressure in model membranes using di-pyrenyl phosphatidylcholine. Faraday Discuss 111:41-53. discussion 69-78

42. Kamo T, Handa T, Nakano M (2013) Lateral pressure change on phase transitions of phosphatidylcholine/diolein mixed membranes, vol 104

43. Orsi M, Noro MG, Essex JW (2011) Dual-resolution molecular dynamics simulation of antimicrobials in biomembranes. J R Soc Interface 6(59):826-841

44. Marsh D (2007) Lateral pressure profile, spontaneous curvature frustration, and the incorporation and conformation of proteins in membranes. Biophys J 93(11):3884-3899

45. van den Brink-van der Laan E, Killian JA, de Kruijff Ben (2004) Nonbilayer lipids affect peripheral and integral membrane proteins via changes in the lateral pressure profile. Biochimica et Biophysica Acta (BBA) - Biomembranes 1666(1-2): 275-288

46. Cantor RS (1997) The lateral pressure profile in membranes: a physical mechanism of general anesthesia. Biochemistry 36(9):2339-2344

47. Ollila S, Hyvönen MT, Vattulainen I (2007) Polyunsaturation in lipid membranes: dynamic properties and lateral pressure profiles. J Phys Chem B 111(12):3139-3150 
48. Ding W, Palaiokostas M, Shahane G, Wang W, Orsi M (2017) Effects of high pressure on phospholipid bilayers. J Phys Chem B 121(41):9597-9606

49. Orsi M, Essex JW (2013) Physical properties of mixed bilayers containing lamellar and nonlamellar lipids: insights from coarse-grain molecular dynamics simulations. Faraday Discuss, $1-24$

50. Ding W, Palaiokostas M, Wang W, Orsi M (2015) Effects of lipid composition on bilayer membranes quantified by All-Atom molecular dynamics. J Phys Chem B 119:15263-15274

51. Perrin SB, Sodt AJ, Cotten ML, Pastor RW (2014) The curvature induction of surface-bound antimicrobial peptides piscidin 1 and piscidin 3 varies with lipid chain length. J Membrane Biol 248(3):455-467

52. Clarke RJ (2001) The dipole potential of phospholipid membranes and methods for its detection. Adv Colloid Interface Sci 89-90:263-281

53. Liberman EA, Topaly VP (1969) Permeability of bimolecular phospholipid membranes for fat-soluble ions. Biofizika 14(3):452-461

54. Starke-Peterkovic T, Turner Nsl, Else PL, Clarke RJ (2005) Electric field strength of membrane lipids from vertebrate species: membrane lipid composition and $\mathrm{Na}+\mathrm{K}+$-ATPase molecular activity. Am J Physiol Regul Integr Comp Physiol 288(3):63-70

55. Starke-Peterkovic T, Clarke RJ (2009) Effect of headgroup on the dipole potential of phospholipid vesicles. Eur Biophys J 39(1):103-110

56. Balleza D, Garcia-Arribas AB, Sot J, Ruiz-Mirazo K, Goni FM (2014) Ether- versus ester-linked phospholipid bilayers containing either linear or branched apolar chains. Biophys $\mathbf{J}$ 107(6):1364-1374

57. Orsi M, Michel J, Essex JW (2010) Coarse-grain modelling of DMPC and DOPC lipid bilayers. J Phys Condens Matter 22(15):155106

58. Horn JN, Romo TD, Grossfield A (2013) Simulating the mechanism of antimicrobial lipopeptides with all-atom molecular dynamics. Biochemistry 52(33):5604-5610

59. MacKerell AD Jr, Bashford D, Bellott M, Dunbrack RL Jr, Evanseck JD, Field MJ, Fischer S, Gao J, Guo H, Ha S, JosephMcCarthy D, Kuchnir L, Kuczera K, Lau FTK, Mattos C, Michnick S, Ngo T, Nguyen DT, Prodhom B, Reiher WE, Roux B, Schlenkrich M, Smith JC, Stote R, Straub J, Watanabe M, Wiórkiewicz-Kuczera J, Yin D, Karplus M (1998) All-Atom empirical potential for molecular modeling and dynamics studies of proteins. J Phys Chem B 102(18):3586-3616

60. Klauda JB, Venable RM, Alfredo FJ, O'Connor JW, Tobias DJ, Mondragon-Ramirez C, Vorobyov I, MacKerell AD Jr, Pastor RW (2010) Update of the CHARMM all-atom additive force field for lipids: validation on six lipid types. J Phys Chem B 114(23):7830-7843

61. Wu EL, Xi C, Jo S, Rui H, Song KC, Dávila-Contreras EM, Qi Y, Lee J, Monje-Galvan V, Venable RM, Klauda JB, Im W (2014) CHARMM-GUI membrane builder toward realistic biological membrane simulations. J Comput Chem 35(27): 1997-2004

62. Jo S, Lim JB, Klauda JB, Im W (2009) CHARMM-GUI membrane builder for mixed bilayers and its application to yeast membranes. Biophys J 97(1):50-58

63. Jo S, Kim T, Im W (2007) Automated builder and database of protein/membrane complexes for molecular dynamics simulations. PLoS One 2(9):880

64. Jo S, Kim T, Iyer VG, Im W (2008) CHARMM-GUI: a Webbased graphical user interface for CHARMM. J Comput Chem 29(11):1859-1865
65. Doktorova M, Weinstein H (2018) Accurate in silico modeling of asymmetric bilayers based on biophysical principles. Biophys J 115(9):1638-1643

66. Van Der Spoel D, Lindahl E, Hess B, Groenhof G, Mark AE, Berendsen HJC (2005) GROMACS: fast, flexible, and free. J Comput Chem 26(16):1701-1718

67. Hess B, Kutzner C, van der Spoel D, Lindahl E (2008) GROMACS 4: algorithms for highly efficient, load-balanced, and scalable molecular simulation. J Chem Theory Comput 4(3):435-447

68. Bussi G, Donadio D, Parrinello M (2007) Canonical sampling through velocity rescaling. J Chem Phys 126(1):014101

69. Venable RM, Brown FLH, Pastor RW (2015) Mechanical properties of lipid bilayers from molecular dynamics simulation. Chem Phys Lipids 192:60-74

70. Siu SWI, Vácha R, Jungwirth P, Böckmann RA (2008) Biomolecular simulations of membranes: physical properties from different force fields, vol 128

71. Shinoda K, Shinoda W, Baba T, Mikami M (2004) Comparative molecular dynamics study of ether- and ester-linked phospholipid bilayers. J Chem Phys 121(19):9648-9654

72. Orsi M (2014) Comparative assessment of the ELBA coarsegrained model for water. Mol Phys 112:1566-1576

73. Berendsen HJC, Postma JPM, van Gunsteren WF, DiNola A, Haak JR (1984) Molecular dynamics with coupling to an external bath. J Chem Phys 81(8):3684-3690

74. Parrinello M, Rahman A (1981) Polymorphic transitions in single crystals: a new molecular dynamics method. J Appl Phys 52(12):7182-7190

75. Shuichi M, Kollman PA (1992) Settle: an analytical version of the shake and rattle algorithm for rigid water models. J Comput Chem 13(8):952-962

76. Berk H, Henk B, Herman BJC, Johannes FGEM (1997) Lincs: a linear constraint solver for molecular simulations. J Comput Chem 18(12):1463-1472

77. Darden T, York D, Pedersen L (1993) Particle mesh Ewald: an $\mathrm{N} \cdot \log (\mathrm{N})$ method for Ewald sums in large systems. J Chem Phys 98(12):10089-10092

78. Lukat G, Krüger J, Sommer B (2013) APL@ Voro: a Voronoibased membrane analysis tool for GROMACS trajectories. J Chem Inf Model 53(11):2908-2925

79. Vanegas JM, Torres-Sánchez A, Arroyo M (2014) Importance of force decomposition for local stress calculations in biomembrane molecular simulations. J Chem Theory Comput 10(2):691702

80. Torres-Sánchez A, Vanegas JM, Arroyo M (2015) Examining the mechanical equilibrium of microscopic stresses in molecular simulations. Phys Rev Lett 114(25):258102

81. Samuli Ollila OH, Jelger Risselada H, Louhivuori M, Lindahl E, Vattulainen I, Marrink SJ (2009) 3D pressure field in lipid membranes and membrane-protein complexes. Phys Rev Lett 102(7):078101

82. Thijsse BJ, Hollanders MA, Hendrikse J (1998) A practical algorithm for least-squares spline approximation of data containing noise. Comput Phys 12(4)

83. McIntosh TJ (1978) The effect of cholesterol on the structure of phosphatidylcholine bilayers. Biochimica et Biophysica Acta (BBA) - Biomembranes 513(1):43-58

84. Hofsäss C, Lindahl E, Edholm O (2003) Molecular dynamics simulations of phospholipid bilayers with cholesterol. Biophys $\mathrm{J}$ 84(4):2192-2206

85. Leftin A, Molugu TR, Job C, Beyer K, Brown MF (2014) Area per lipid and cholesterol interactions in membranes from separated local-field 13C NMR spectroscopy. Biophys J 107(10):2274-2286 
86. de Meyer F, Smit B (2009) Effect of cholesterol on the structure of a phospholipid bilayer. Proc Natl Acad Sci USA 106(10):3654-3658

87. Kučerka N, Pencer J, Nieh M-P, Katsaras J (2007) Influence of cholesterol on the bilayer properties of monounsaturated phosphatidylcholine unilamellar vesicles. Eur Phys J E Soft Matter 23(3):247-254

88. Asawakarn T, Cladera J, O'Shea P (2001) Effects of the membrane dipole potential on the interaction of saquinavir with phospholipid membranes and plasma membrane receptors of Caco-2 cells. J Biol Chem 276(42):38457-38463

89. Lipowsky R, Sackmann E (1995) Structure and dynamics of membranes. Number v. 1, pt. 1 in Handbook of biological physics. Elsevier Science

90. Mukhin SI, Baoukina S (2005) Analytical derivation of thermodynamic characteristics of lipid bilayer from a flexible string model. Phys Rev E 71(6):061918

91. Srinivas Reddy A, Warshaviak DT, Chachisvilis M (2012) Effect of membrane tension on the physical properties of DOPC lipid bilayer membrane. Biochim Biophys Acta Biomembr 1818(9):2271-2281

92. Zhao W, Ròg T, Gurtovenko AA, Vattulainen I, Karttunen M (2007) Atomic-scale structure and electrostatics of anionic palmitoyloleoylphosphatidylglycerol lipid bilayers with $\mathrm{Na}+$ counterions. Biophys J 92(4):1114-24

93. Kuvcerka N, Nieh M-P, Katsaras J (2011) Fluid phase lipid areas and bilayer thicknesses of commonly used phosphatidylcholines as a function of temperature. Biochimica et Biophysica Acta (BBA) - Biomembranes 1808(11):2761-2771

94. Kucerka N, Tristram-Nagle S, Nagle JF (2005) Structure of fully hydrated fluid phase lipid bilayers with monounsaturated chains. J Membr Biol 208(3):193-202

95. Skjevik ÅA, Madej BD, Dickson CJ, Teigen K, Walker RC, Gould IR (2015) All-atom lipid bilayer self-assembly with the AMBER and CHARMM lipid force fields. Chem Commun 51(21):4402-4405

96. Rand RP, Fuller N, Parsegian VA, Rau DC (1988) Variation in hydration forces between neutral phospholipid bilayers: evidence for hydration attraction. Biochemistry 27(20):7711-7722

97. Rappolt M, Hickel A, Bringezu F, Lohner K (2003) Mechanism of the lamellar/inverse hexagonal phase transition examined by high-resolution X-ray diffraction. Biophys J 84(5):31113122
98. Kuvčerka N, Holland BW, Gray CG, Tomberli B, Katsaras J (2012) Scattering density profile model of POPG bilayers as determined by molecular dynamics simulations and smallangle neutron and X-ray scattering experiments. J Phys Chem B 116(1):232-239

99. Tolokh IS, Vivcharuk V, Tomberli B, Gray CG (2009) Binding free energy and counterion release for adsorption of the antimicrobial peptide lactoferricin B on a POPG membrane. Phys Rev E 80(3):031911

100. Pan J, Heberle FA, Tristram-Nagle S, Szymanski M, Koepfinger M, Katsaras J, Kučerka N (2012) Molecular structures of fluid phase phosphatidylglycerol bilayers as determined by smallangle neutron and X-ray scattering. Biochimica et Biophysica Acta (BBA) - Biomembranes 1818(9):2135-2148

101. Skjevik ÅA, Madej BD, Dickson CJ, Lin C, Teigen K, Walker RC, Gould IR (2016) Simulation of lipid bilayer self-assembly using all-atom lipid force fields. Phys Chem Chem Phys: PCCP 18(15):10573-10584

102. Pan J, Cheng X, Monticelli L, Heberle FA, Kučerka N, Tieleman DP, Katsaras J (2014) The molecular structure of a phosphatidylserine bilayer determined by scattering and molecular dynamics simulations. Soft Matter 10(21):3716-3725

103. Mukhopadhyay P, Monticelli L, Tieleman DP (2004) Molecular dynamics simulation of a palmitoyl-oleoyl phosphatidylserine bilayer with na+ counterions and $\mathrm{NaCl}$. Biophys J 86(3):16011609

104. Olsen BN, Bielska AA, Lee T, Daily MD, Covey DF, Schlesinger PH, Baker NA, Ory DS (2013) The structural basis of cholesterol accessibility in membranes. Biophys J 105(8):1838-1847

105. Loura LMS, do Canto AMTM, Martins J (2013) Sensing hydration and behavior of pyrene in POPC and POPC/cholesterol bilayers: a molecular dynamics study. Biochimica et Biophysica Acta (BBA) - Biomembranes 1828(3):1094-1101

106. Hong C, Peter Tieleman D, Yi W (2014) Microsecond molecular dynamics simulations of lipid mixing. Langmuir 30(40):1199312001

107. Murzyn K, Róg T (2005) Marta Pasenkiewicz-Gierula phosphatidylethanolamine-phosphatidylglycerol bilayer as a model of the inner bacterial membrane. Biophys $J$ 88(2): 1091-1103

108. Humphrey W, Dalke A, Schulten K (1996) VMD: visual molecular dynamics. J Mol Graph 14(1):33-8 